\title{
An implicit iterative algorithm with errors for two families of generalized asymptotically nonexpansive mappings
}

\author{
Ravi P Agarwal ${ }^{1}$, Xiaolong Qin² and Shin Min Kang ${ }^{3 *}$
}

\footnotetext{
* Correspondence: smkang@gnu. ac.kr

${ }^{3}$ Department of Mathematics and RINS, Gyeongsang National University, Jinju 660-701, Korea Full list of author information is available at the end of the article
}

\begin{abstract}
In this paper, an implicit iterative algorithm with errors is considered for two families of generalized asymptotically nonexpansive mappings. Strong and weak convergence theorems of common fixed points are established based on the implicit iterative algorithm.

Mathematics Subject Classification (2000) 47H09 · 47H10 · 47J25

Keywords: Asymptotically nonexpansive mapping, common fixed point, implicit iterative algorithm, generalized asymptotically nonexpansive mapping
\end{abstract}

\section{Introduction}

In nonlinear analysis theory, due to applications to complex real-world problems, a growing number of mathematical models are built up by introducing constraints which can be expressed as subproblems of a more general problem. These constraints can be given by fixed-point problems, see, for example, [1-3]. Study of fixed points of nonlinear mappings and its approximation algorithms constitutes a topic of intensive research efforts. Many well-known problems arising in various branches of science can be studied by using algorithms which are iterative in their nature. The well-known convex feasibility problem which captures applications in various disciplines such as image restoration, computer tomography, and radiation therapy treatment planning is to find a point in the intersection of common fixed point sets of a family of nonexpansive mappings, see, for example, [3-5].

For iterative algorithms, the most oldest and simple one is Picard iterative algorithm. It is known that $T$ enjoys a unique fixed point, and the sequence generated in Picard iterative algorithm can converge to the unique fixed point. However, for more general nonexpansive mappings, Picard iterative algorithm fails to convergence to fixed points of nonexpansive even that it enjoys a fixed point.

Recently, Mann-type iterative algorithm and Ishikawa-type iterative algorithm (implicit and explicit) have been considered for the approximation of common fixed points of nonlinear mappings by many authors, see, for example, [6-24]. A classical convergence theorem of nonexpansive mappings has been established by $\mathrm{Xu}$ and Ori [23]. In 2006, Chang et al. [6] considered an implicit iterative algorithm with errors for asymptotically nonexpansive mappings in a Banach space. Strong and weak convergence theorems are

(c) 2011 Agarwal et al; licensee Springer. This is an Open Access article distributed under the terms of the Creative Commons Attribution License (http://creativecommons.org/licenses/by/2.0), which permits unrestricted use, distribution, and reproduction in any medium, provided the original work is properly cited. 
established. Recently, Cianciaruso et al. [9] considered an Ishikawa-type iterative algorithm for the class of asymptotically nonexpansive mappings. Strong and weak convergence theorems are also established. In this paper, based on the class of generalized asymptotically nonexpansive mappings, an Ishikawa-type implicit iterative algorithm with errors for two families of mappings is considered. Strong and weak convergence theorems of common fixed points are established. The results presented in this paper mainly improve the corresponding results announced in Chang et al. [6], Chidume and Shahzad [7], Cianciaruso et al. [9], Guo and Cho [10], Khan et al. [12], Plubtieng et al. [14], Qin et al. [15], Shzhzad and Zegeye [18], Thakur [21], Thianwan and Suantai [22], $\mathrm{Xu}$ and Ori [23], Zhou and Chang [24].

\section{Preliminaries}

Let $C$ be a nonempty closed convex subset of a Banach space $E$. Let $T: C \rightarrow C$ be a mapping. Throughout this paper, we use $F(T)$ to denote the fixed point set of $T$.

Recall the following definitions.

$T$ is said to be nonexpansive if

$$
\|T x-T y\| \leq\|x-y\|, \quad \forall x, y \in C .
$$

$T$ is said to be asymptotically nonexpansive if there exists a positive sequence $\left\{h_{n}\right\} \subset$ $[1, \infty)$ with $h_{n} \rightarrow 1$ as $n \rightarrow \infty$ such that

$$
\left\|T^{n} x-T^{n} y\right\| \leq h_{n}\|x-y\|, \quad \forall x, y \in C, n \geq 1 .
$$

It is easy to see that every nonexpansive mapping is asymptotically nonexpansive with the asymptotical sequence $\{1\}$. The class of asymptotically nonexpansive mappings was introduced by Goebel and Kirk [25] in 1972. It is known that if $C$ is a nonempty bounded closed convex subset of a uniformly convex Banach space $E$, then every asymptotically nonexpansive mapping on $C$ has a fixed point. Further, the set $F(T)$ of fixed points of $T$ is closed and convex. Since 1972, a host of authors have studied weak and strong convergence problems of implicit iterative processes for such a class of mappings.

$T$ is said to be asymptotically nonexpansive in the intermediate sense if it is continuous and the following inequality holds:

$$
\limsup _{n \rightarrow \infty} \sup _{x, y \in C}\left(\left\|T^{n} x-T^{n} y\right\|-\|x-y\|\right) \leq 0 .
$$

Putting $\xi_{n}=\max \left\{0, \sup _{x, y \in C}\left(\left\|T^{n} x-T^{n} y\right\|-\|x-y\|\right)\right\}$, we see that $\xi_{n} \rightarrow 0$ as $n \rightarrow \infty$. Then, (2.1) is reduced to the following:

$$
\left\|T^{n} x-T^{n} y\right\| \leq\|x-y\|+\xi_{n}, \quad \forall x, y \in C, n \geq 1 .
$$

The class of asymptotically nonexpansive mappings in the intermediate sense was introduced by Bruck et al. [26] (see also Kirk [27]) as a generalization of the class of asymptotically nonexpansive mappings. It is known that if $C$ is a nonempty closed convex and bounded subset of a real Hilbert space, then every asymptotically nonexpansive self mapping in the intermediate sense has a fixed point; see [28] more details.

$T$ is said to be generalized asymptotically nonexpansive if it is continuous and there exists a positive sequence $\left\{h_{n}\right\} \subset[1, \infty)$ with $h_{n} \rightarrow 1$ as $n \rightarrow \infty$ such that 


$$
\limsup _{n \rightarrow \infty} \sup _{x, y \in C}\left(\left\|T^{n} x-T^{n} y\right\|-h_{n}\|x-y\|\right) \leq 0
$$

Putting $\xi_{n}=\max \left\{0, \sup _{x, y \in C}\left(|| T^{n} x-T^{n} y||-h_{n}|| x-y||\right)\right\}$, we see that $\xi_{n} \rightarrow 0$ as $n \rightarrow$ $\infty$. Then, (2.2) is reduced to the following:

$$
\left\|T^{n} x-T^{n} y\right\| \leq h_{n}\|x-y\|+\xi_{n}, \quad \forall x, y \in C, n \geq 1 .
$$

We remark that if $h_{n} \equiv 1$, then the class of generalized asymptotically nonexpansive mappings is reduced to the class of asymptotically nonexpansive mappings in the intermediate.

In 2006, Chang et al. [6] considered the following implicit iterative algorithms for a finite family of asymptotically nonexpansive mappings $\left\{T_{1}, T_{2}, \ldots, T_{N}\right\}$ with $\left\{\alpha_{n}\right\}$ a real sequence in $(0,1),\left\{u_{n}\right\}$ a bounded sequence in $C$ and an initial point $x_{0} \in C$ :

$$
\begin{aligned}
x_{1}= & \alpha_{1} x_{0}+\left(1-\alpha_{1}\right) T_{1} x_{1}+u_{1}, \\
x_{2}= & \alpha_{2} x_{1}+\left(1-\alpha_{2}\right) T_{2} x_{2}+u_{2}, \\
& \cdots \\
x_{N}= & \alpha_{N} x_{N-1}+\left(1-\alpha_{N}\right) T_{N} x_{N}+u_{N \prime}, \\
x_{N+1}= & \alpha_{N+1} x_{N}+\left(1-\alpha_{N+1}\right) T_{1}^{n} x_{N+1}+u_{N+1}, \\
& \cdots \\
x_{2 N}= & \alpha_{2 N} x_{2 N-1}+\left(1-\alpha_{2 N}\right) T_{N}^{2} x_{2 N}+u_{2 N}, \\
x_{2 N+1}= & \alpha_{2 N+1} x_{2 N}+\left(1-\alpha_{2 N+1}\right) T_{1}^{3} x_{2 N+1}+u_{2 N+1}, \\
& \cdots .
\end{aligned}
$$

The above table can be rewritten in the following compact form:

$$
x_{n}=\alpha_{n} x_{n-1}+\left(1-\alpha_{n}\right) T_{i(n)}^{j(n)} x_{n}+u_{n}, \quad \forall n \geq 1,
$$

where for each $n \geq 1$ fixed, $j(n)$ - 1 denotes the quotient of the division of $n$ by $N$ and $i(n)$ the rest, i.e., $n=(j(n)-1) N+i(n)$.

Based on the implicit iterative algorithm, they obtained, under the assumption that $C+$ $C \subset C$, weak and strong convergence theorems of common fixed points for a finite family of asymptotically nonexpansive mappings $\left\{T_{1}, T_{2}, \ldots, T_{N}\right\}$; see [6] for more details.

Recently, Cianciaruso et al. [9] considered a Ishikawa-like iterative algorithm for the class of asymptotically nonexpansive mappings in a Banach space. To be more precise, they introduced and studied the following implicit iterative algorithm with errors.

$$
\left\{\begin{array}{l}
y_{n}=\left(1-\beta_{n}-\delta_{n}\right) x_{n}+\beta_{n} T_{i(n)}^{j(n)} x_{n}+\delta_{n} v_{n}, \\
x_{n}=\left(1-\alpha_{n}-\gamma_{n}\right) x_{n-1}+\alpha_{n} T_{i(n)}^{j(n)} y_{n}+\gamma_{n} u_{n}, \quad \forall n \geq 1,
\end{array}\right.
$$

where $\left\{\alpha_{n}\right\},\left\{\beta_{n}\right\},\left\{\gamma_{n}\right\}$, and $\left\{\delta_{n}\right\}$ are real number sequences in $[0,1],\left\{u_{n}\right\}$ and $\left\{v_{n}\right\}$ are bounded sequence in $C$. Weak and strong convergence theorems are established in a uniformly convex Banach space; see [29] for more details.

In this paper, motivated and inspired by the results announced in Chang et al. [6], Chidume and Shahzad [7], Cianciaruso et al. [9], Guo and Cho [10], Plubtieng et al. [14], Qin et al. [15], Shzhzad and Zegeye [18], Thakur [21], Thianwan and Suantai [22], $\mathrm{Xu}$ and Ori [23], Zhou and Chang [24], we consider the following Ishikawa-like implicit iteration algorithm with errors for two finite families of generalized asymptotically nonexpansive mappings $\left\{T_{1}, T_{2}, \ldots, T_{N}\right\}$ and $\left\{S_{1}, S_{2}, \ldots, S_{N}\right\}$. 


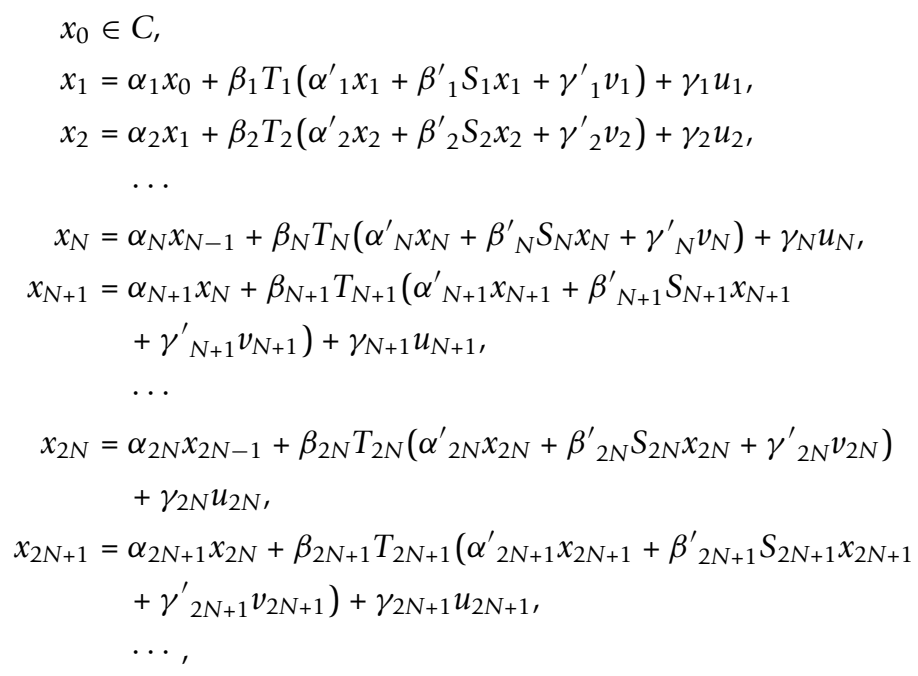

where $\left\{\alpha_{n}\right\},\left\{\beta_{n}\right\},\left\{\gamma_{n}\right\},\left\{\alpha_{n}^{\prime}\right\},\left\{\beta_{n}^{\prime}\right\}$, and $\left\{\gamma_{n}^{\prime}\right\}$ are sequences in $[0,1]$ such that $\alpha_{n}+\beta_{n}+\gamma_{n}=\alpha_{n}^{\prime}+\beta_{n}^{\prime}+\gamma_{n}^{\prime}=1$ for each $n \geq 1$. We have rewritten the above table in the following compact form:

$$
x_{n}=\alpha_{n} x_{n-1}+\beta_{n} T_{i(n)}^{j(n)}\left(\alpha_{n}^{\prime} x_{n}+\beta_{n}^{\prime} S_{i(n)}^{j(n)} x_{n}+\gamma_{n}^{\prime} v_{n}\right)+\gamma_{n} u_{n}, \quad n \geq 1,
$$

where for each $n \geq 1$ fixed, $j(n)-1$ denotes the quotient of the division of $n$ by $N$ and $i(n)$ the rest, i.e., $n=(j(n)-1) N+i(n)$.

Putting $y_{n}=\alpha_{n}^{\prime} x_{n}+\beta_{n}^{\prime} S_{n} x_{n}+\gamma_{n}^{\prime} v_{n}$, we have the following composite iterative algorithm:

$$
\left\{\begin{array}{l}
y_{n}=\alpha^{\prime}{ }_{n} x_{n}+\beta^{\prime}{ }_{n} S_{i(n)}^{j(n)} x_{n}+\gamma^{\prime}{ }_{n} v_{n} \\
x_{n}=\alpha_{n} x_{n-1}+\beta_{n} T_{i(n)}^{j(n)} y_{n}+\gamma_{n} u_{n}, \quad n \geq 1 .
\end{array}\right.
$$

We remark that the implicit iterative algorithm (2.4) is general which includes (2.3) as a special case.

Now, we show that (2.4) can be employed to approximate fixed points of generalized asymptotically nonexpansive mappings which is assumed to be Lipschitz continuous. Let $T_{i}$ be a $L_{t}^{i}$-Lipschitz generalized asymptotically nonexpansive mapping with a sequence $\left\{h_{n}^{i}\right\} \subset[1, \infty)$ such that $h_{n}^{i} \rightarrow 1$ as $n \rightarrow \infty$ and $S_{i}$ be a $L_{s}^{i}$-Lipschitz generalized asymptotically nonexpansive mapping with sequences $\left\{k_{n}^{i}\right\} \subset[1, \infty)$ such that $k_{n}^{i} \rightarrow 1$ as $n \rightarrow \infty$ for each $1 \leq i \leq N$. Define a mapping $W_{n}: C \rightarrow C$ by

$$
W_{n}(x)=\alpha_{n} x_{n-1}+\beta_{n} T_{i(n)}^{j(n)}\left(\alpha_{n}^{\prime} x+\beta_{n}^{\prime} S_{i(n)}^{j(n)} x+\gamma_{n}^{\prime} v_{n}\right)+\gamma_{n} u_{n}, \quad \forall n \geq 1 .
$$

It follows that

$$
\begin{aligned}
& \left\|W_{n}(x)-W_{n}(y)\right\| \\
& \leq \beta_{n}\left\|T_{i(n)}^{j(n)}\left(\alpha^{\prime}{ }_{n} x+\beta^{\prime}{ }_{n} S_{i(n)}^{j(n)} x+\gamma^{\prime}{ }_{n} v_{n}\right)-T_{i(n)}^{j(n)}\left(\alpha^{\prime}{ }_{n} y+\beta^{\prime}{ }_{n} S_{i(n)}^{j(n)} y+\gamma^{\prime}{ }_{n} v_{n}\right)\right\| \\
& \leq \beta_{n} L\left(\alpha^{\prime}{ }_{n}\|x-y\|+\beta^{\prime}{ }_{n}\left\|S_{i(n)}^{j(n)} x-S_{i(n)}^{j(n)} y\right\|\right) \\
& \leq \beta_{n} L\left(\alpha^{\prime}{ }_{n}+\beta^{\prime}{ }_{n} L\right)\|x-y\|, \quad \forall x, y \in C,
\end{aligned}
$$


where

$$
L=\max \left\{L_{t}^{1}, \ldots, L_{t}^{N}, L_{s}^{1}, \ldots L_{s}^{N}\right\} .
$$

If $\beta_{n} L\left(\alpha_{n}^{\prime}+\beta_{n}^{\prime} L\right)<1$ for all $n \geq 1$, then $W_{n}$ is a contraction. By Banach contraction mapping principal, we see that there exists a unique fixed point $x_{n} \in C$ such that

$$
\begin{aligned}
x_{n}=W_{n}\left(x_{n}\right)= & \alpha_{n} x_{n-1}+\beta_{n} T_{i(n)}^{j(n)}\left(\alpha^{\prime}{ }_{n} x+\beta^{\prime}{ }_{n} S_{i(n)}^{j(n)} x+\gamma^{\prime}{ }_{n} v_{n}\right) \\
& +\gamma_{n} u_{n}, \quad \forall n \geq 1 .
\end{aligned}
$$

That is, the implicit iterative algorithm (2.4) is well defined.

The purpose of this paper is to establish strong and weak convergence theorem of fixed points of generalized asymptotically nonexpansive mappings based on (2.4).

Next, we recall some well-known concepts.

Let $E$ be a real Banach space and $U_{E}=\{x \in E:\|x\|=1\}$. $E$ is said to be uniformly convex if for any $\varepsilon \in(0,2]$ there exists $\delta>0$ such that for any $x, y \in U_{E}$,

$$
\|x-y\| \geq \varepsilon \quad \text { implies } \quad\left\|\frac{x+y}{2}\right\| \leq 1-\delta .
$$

It is known that a uniformly convex Banach space is reflexive and strictly convex.

Recall that $E$ is said to satisfy Opial's condition [30] if for each sequence $\left\{x_{n}\right\}$ in $E$, the condition that the sequence $x_{n} \rightarrow x$ weakly implies that

$$
\liminf _{n \rightarrow \infty}\left\|x_{n}-x\right\|<\liminf _{n \rightarrow \infty}\left\|x_{n}-y\right\|
$$

for all $y \in E$ and $y \neq x$. It is well known [30] that each $l^{p}(1 \leq p<\infty)$ and Hilbert spaces satisfy Opial's condition. It is also known [29] that any separable Banach space can be equivalently renormed to that it satisfies Opial's condition.

Recall that a mapping $T: C \rightarrow C$ is said to be demiclosed at the origin if for each sequence $\left\{x_{n}\right\}$ in $C$, the condition $x_{n} \rightarrow x_{0}$ weakly and $T x_{n} \rightarrow 0$ strongly implies $T x_{0}=$ 0 . $T$ is said to be semicompact if any bounded sequence $\left\{x_{n}\right\}$ in $C$ satisfying $\lim _{n \rightarrow \infty} \| x_{n}$ $-T x_{n} \|=0$ has a convergent subsequence.

In order to prove our main results, we also need the following lemmas.

Lemma 2.1. [20] Let $\left\{a_{n}\right\},\left\{b_{n}\right\}$ and $\left\{c_{n}\right\}$ be three nonnegative sequences satisfying the following condition:

$$
a_{n+1} \leq\left(1+b_{n}\right) a_{n}+c_{n}, \quad \forall n \geq n_{0},
$$

where $n_{0}$ is some nonnegative integer. If $\sum_{n=0}^{\infty} c_{n}<\infty$ and $\sum_{n=0}^{\infty} b_{n}<\infty$, then $\lim _{n \rightarrow \infty}$ $a_{n}$ exists.

Lemma 2.2. [17]Let $E$ be a uniformly convex Banach space and $0<\lambda \leq t_{n} \leq \eta<1$ for all $n \geq 1$. Suppose that $\left\{x_{n}\right\}$ and $\left\{y_{n}\right\}$ are sequences of $E$ such that

$$
\limsup _{n \rightarrow \infty}\left\|x_{n}\right\| \leq r, \quad \limsup _{n \rightarrow \infty}\left\|y_{n}\right\| \leq r
$$

and

$$
\lim _{n \rightarrow \infty}\left\|t_{n} x_{n}+\left(1-t_{n}\right) y_{n}\right\|=r
$$

hold for some $r \geq 0$. Then $\lim _{n \rightarrow \infty}|| x_{n}-y_{n} \|=0$. 
The following lemma can be obtained from Qin et al. [31] or Sahu et al. [32] immediately.

Lemma 2.3. Let $C$ be a nonempty closed convex subset of a real Hilbert space H. Let $T: C \rightarrow C$ be a Lipschitz generalized asymptotically nonexpansive mapping. Then $I-T$ is demiclosed at origin.

\section{Main results}

Now, we are ready to give our main results in this paper.

Theorem 3.1. Let $E$ be a real uniformly convex Banach space and $C$ be a nonempty closed convex subset of E. Let $T_{i}: C \rightarrow C$ be a uniformly $L_{t}^{i}$-Lipschitz and generalized asymptotically nonexpansive mapping with a sequence $\left\{h_{n}^{i}\right\} \subset[1, \infty)$, where $h_{n}^{i} \rightarrow 1$ as $n$ $\rightarrow \infty$ and $S_{i}: C \rightarrow C$ be a uniformly $L_{s}^{i}$-Lipschitz and generalized asymptotically nonexpansive mapping with a sequence $\left\{k_{n}^{i}\right\} \subset[1, \infty)$, where $k_{n}^{i} \rightarrow 1$ as $n \rightarrow \infty$ for each $1 \leq i$ $\leq N$. Assume that $\mathcal{F}=\bigcap_{i=1}^{N} F\left(T_{i}\right) \bigcap \bigcap_{i=1}^{N} F\left(S_{i}\right) \neq \emptyset$. Let $\left\{u_{n}\right\},\left\{v_{n}\right\}$ be bounded sequences in $C$ and $e_{n}=\max \left\{h_{n}, k_{n}\right\}$, where $h_{n}=\sup \left\{h_{n}^{i}: 1 \leq i \leq N\right\}$ and $k_{n}=\sup \left\{k_{n}^{i}: 1 \leq i \leq N\right\}$. Let $\left\{\alpha_{n}\right\},\left\{\beta_{n}\right\},\left\{\gamma_{n}\right\},\left\{\alpha_{n}^{\prime}\right\},\left\{\beta_{n}^{\prime}\right\}$ and $\left\{\gamma_{n}^{\prime}\right\}$ be sequences in $[0,1]$ such that $\alpha_{n}+\beta_{n}+\gamma_{n}=\alpha_{n}^{\prime}+\beta_{n}^{\prime}+\gamma_{n}^{\prime}=1$ for each $n \geq 1$. Let $\left\{x_{n}\right\}$ be a sequence generated in (2.4). Put $\quad \mu_{n}^{i}=\max \left\{0, \sup _{x, y \in C}\left(\left\|T_{i}^{n} x-T_{i}^{n} y\right\|-h_{n}^{i}\|x-y\|\right)\right\}$ and $v_{n}^{i}=\max \left\{0, \sup _{x, y \in C}\left(\left\|S_{i}^{n} x-S_{i}^{n} y\right\|-k_{n}^{i}\|x-y\|\right)\right\}$. Let $\xi_{n}=\max \left\{\mu_{n}, v_{n}\right\}$, where $v_{n}=\max \left\{v_{n}^{i}: 1 \leq i \leq N\right\}$ and $v_{n}=\max \left\{v_{n}^{i}: 1 \leq i \leq N\right\}$. Assume that the following restrictions are satisfied:

(a) $\sum_{n=1}^{\infty} \gamma_{n}<\infty$ and $\sum_{n=1}^{\infty} \gamma_{n}^{\prime}<\infty$;

(b) $\sum_{n=1}^{\infty}\left(e_{n}-1\right)<\infty$ and $\sum_{n=1}^{\infty} \xi_{n}<\infty$;

(c) $\beta_{n} L\left(\alpha_{n}^{\prime}+\beta_{n}^{\prime} L\right)<1$, where $L$ is defined in (2.5);

(d) there exist constants $\lambda, \eta \in(0,1)$ such that $\lambda \leq \alpha_{n}, \alpha_{n}^{\prime} \leq \eta$.

Then

$$
\lim _{n \rightarrow \infty}\left\|x_{n}-T_{r} x_{n}\right\|=\lim _{n \rightarrow \infty}\left\|x_{n}-S_{r} x_{n}\right\|=0, \quad \forall r \in\{1,2, \ldots, N\} .
$$

Proof. Fixing $f \in \mathcal{F}$, we see that

$$
\begin{aligned}
\left\|y_{n}-f\right\| & =\left\|\alpha^{\prime}{ }_{n} x_{n}+\beta^{\prime}{ }_{n} S_{i(n)}^{j(n)} x_{n}+\gamma^{\prime}{ }_{n} v_{n}-f\right\| \\
& \leq \alpha^{\prime}{ }_{n}\left\|x_{n}-f\right\|+\beta^{\prime}{ }_{n}\left\|S_{i(n)}^{j(n)} x_{n}-f\right\|+\gamma^{\prime}{ }_{n}\left\|v_{n}-f\right\| \\
& \leq \alpha^{\prime}{ }_{n}\left\|x_{n}-f\right\|+\beta^{\prime}{ }_{n} e_{j(n)}\left\|x_{n}-f\right\|+\beta^{\prime}{ }_{n} \xi_{j(n)}+\gamma^{\prime}{ }_{n}\left\|v_{n}-f\right\| \\
& \leq e_{j(n)}\left\|x_{n}-f\right\|+\beta^{\prime}{ }_{n} \xi_{j(n)}+\gamma^{\prime}{ }_{n}\left\|v_{n}-f\right\|
\end{aligned}
$$

and

$$
\begin{aligned}
& \left\|x_{n}-f\right\| \\
& =\left\|\alpha_{n} x_{n-1}+\beta_{n} T_{i(n)}^{j(n)} y_{n}+\gamma_{n} u_{n}-f\right\| \\
& \leq \alpha_{n}\left\|x_{n-1}-f\right\|+\beta_{n}\left\|T_{i(n)}^{j(n)} y_{n}-f\right\|+\gamma_{n}\left\|u_{n}-f\right\| \\
& \leq \alpha_{n}\left\|x_{n-1}-f\right\|+\beta_{n} e_{j(n)}\left\|y_{n}-f\right\|+\beta_{n} \xi_{j(n)}+\gamma_{n}\left\|u_{n}-f\right\| . \\
& \leq \alpha_{n}\left\|x_{n-1}-f\right\|+\left(1-\alpha_{n}\right) e_{j(n)}\left\|y_{n}-f\right\|+\beta_{n} \xi_{j(n)}+\gamma_{n}\left\|u_{n}-f\right\| .
\end{aligned}
$$


Substituting (3.1) into (3.2), we see that

$$
\begin{aligned}
& \left\|x_{n}-f\right\| \\
& \leq \alpha_{n}\left\|x_{n-1}-f\right\|+\left(1-\alpha_{n}\right) e_{j(n)}\left(e_{j(n)}\left\|x_{n}-f\right\|+\beta^{\prime}{ }_{n} \xi_{j(n)}+\gamma^{\prime}{ }_{n}\left\|v_{n}-f\right\|\right) \\
& \quad+\beta_{n} \xi_{j(n)}+\gamma_{n}\left\|u_{n}-f\right\| . \\
& \leq \alpha_{n}\left\|x_{n-1}-f\right\|+\left(1-\alpha_{n}\right) e_{j(n)}^{2}\left\|x_{n}-f\right\|+\left(1+e_{j(n)}\right) \xi_{j(n)} \\
& \quad+e_{j(n)} \gamma^{\prime}{ }_{n}\left\|v_{n}-f\right\|+\gamma_{n}\left\|u_{n}-f\right\| .
\end{aligned}
$$

Notice that $\sum_{n=1}^{\infty}\left(e_{n}-1\right)<\infty$. We see from the restrictions (b) and (d) that there exists a positive integer $n_{0}$ such that

$$
\left(1-\alpha_{n}\right) e_{j(n)}^{2} \leq R<1, \quad \forall n \geq n_{0},
$$

where $R=(1-\lambda)\left(1+\frac{\lambda}{2-2 \lambda}\right)$. It follows that

$$
\begin{aligned}
\left\|x_{n}-f\right\| \leq & \left(1+\frac{\left(1-\alpha_{n}\right)\left(e_{j(n)}^{2}-1\right)}{1-\left(1-\alpha_{n}\right) e_{j(n)}^{2}}\right)\left\|x_{n-1}-f\right\| \\
& +\frac{\left(1+e_{j(n)}\right) \xi_{j(n)}+e_{j(n)} \gamma_{n}^{\prime}\left\|v_{n}-f\right\|+\gamma_{n}\left\|u_{n}-f\right\|}{1-\left(1-\alpha_{n}\right) e_{j(n)}^{2}} \\
\leq & \left(1+\frac{\left(1+M_{1}\right)\left(e_{j(n)}-1\right)}{1-R}\right)\left\|x_{n-1}-f\right\| \\
& +\frac{\left(1+M_{1}\right) \xi_{j(n)}+M_{1} M_{2} \gamma_{n}{ }_{n}+M_{3} \gamma_{n}}{1-R},
\end{aligned}
$$

where $M_{1}=\sup _{n \geq 1}\left\{e_{n}\right\}, M_{2}=\sup _{n \geq 1}\left\{\| v_{n}-f||\right\}$, and $M_{3}=\sup _{n \geq 1}\left\{|| u_{n}-f \mid\right\}$. In view of Lemma 2.1, we see that $\lim _{n \rightarrow \infty}|| x_{n}-f \|$ exists for each $f \in \mathcal{F}$. This implies that the sequence $\left\{x_{n}\right\}$ is bounded. Next, we assume that $\lim _{n \rightarrow \infty}\left\|x_{n}-f\right\|=d>0$. From (3.1), we see that

$$
\begin{aligned}
& \left\|T_{i(n)}^{j(n)} y_{n}-f+\gamma_{n}\left(u_{n}-T_{i(n)}^{j(n)} y_{n}\right)\right\| \\
\leq & \left\|T_{i(n)}^{j(n)} y_{n}-f\right\|+\gamma_{n}\left\|u_{n}-T_{i(n)}^{j(n)} y_{n}\right\| \\
\leq & e_{j(n)}\left\|y_{n}-f\right\|+\xi_{j(n)}+\gamma_{n}\left\|u_{n}-T_{i(n)}^{j(n)} y_{n}\right\| \\
\leq & e_{j(n)}^{2}\left\|x_{n}-f\right\|+e_{j(n)} \xi_{j(n)}+e_{j(n)} \gamma_{n}^{\prime}\left\|v_{n}-f\right\|+\xi_{j(n)} \\
& \quad+\gamma_{n}\left\|u_{n}-T_{i(n)}^{j(n)} y_{n}\right\| .
\end{aligned}
$$

This implies from the restrictions (a) and (b) that

$$
\limsup _{n \rightarrow \infty}\left\|T_{i(n)}^{j(n)} \gamma_{n}-f+\gamma_{n}\left(u_{n}-T_{i(n)}^{j(n)} y_{n}\right)\right\| \leq d .
$$

Notice that

$$
\left\|x_{n-1}-f+\gamma_{n}\left(u_{n}-T_{i(n)}^{j(n)} y_{n}\right)\right\| \leq\left\|x_{n-1}-f\right\|+\gamma_{n}\left\|u_{n}-T_{i(n)}^{j(n)} y_{n}\right\| .
$$

This shows from the restriction (a) that

$$
\limsup _{n \rightarrow \infty}\left\|x_{n-1}-f+\gamma_{n}\left(u_{n}-T_{i(n)}^{j(n)} y_{n}\right)\right\| \leq d .
$$


On the other hand, we have

$$
\begin{aligned}
d= & \lim _{n \rightarrow \infty}\left\|x_{n}-f\right\| \\
= & \lim _{n \rightarrow \infty} \| \alpha_{n}\left(x_{n-1}-f+\gamma_{n}\left(u_{n}-T_{i(n)}^{j(n)} y_{n}\right)\right) \\
& +\left(1-\alpha_{n}\right)\left(T_{i(n)}^{j(n)} y_{n}-f+\gamma_{n}\left(u_{n}-T_{i(n)}^{j(n)} y_{n}\right)\right) \| .
\end{aligned}
$$

It follows from Lemma 2.2 that

$$
\lim _{n \rightarrow \infty}\left\|T_{i(n)}^{j(n)} y_{n}-x_{n-1}\right\|=0
$$

Notice that

$$
\left\|x_{n}-x_{n-1}\right\| \leq \beta_{n}\left\|T_{i(n)}^{j(n)} y_{n}-x_{n-1}\right\|+\gamma_{n}\left\|u_{n}-x_{n-1}\right\| .
$$

It follows from (3.4) and the restriction (a) that

$$
\lim _{n \rightarrow \infty}\left\|x_{n}-x_{n-1}\right\|=0 \text {. }
$$

This implies that

$$
\lim _{n \rightarrow \infty}\left\|x_{n}-x_{n+l}\right\|=0, \quad \forall l=1,2, \ldots, N .
$$

Notice that

$$
\left\|x_{n}-f+\gamma_{n}^{\prime}\left(v_{n}-S_{i(n)}^{j(n)} x_{n}\right)\right\| \leq\left\|x_{n}-f\right\|+\gamma_{n}^{\prime}\left\|v_{n}-S_{i(n)}^{j(n)} x_{n}\right\|
$$

and

$$
\begin{aligned}
& \left\|S_{i(n)}^{j(n)} x_{n}-f+\gamma^{\prime}{ }_{n}\left(v_{n}-S_{i(n)}^{j(n)} x_{n}\right)\right\| \\
& \leq\left\|S_{i(n)}^{j(n)} x_{n}-f\right\|+\gamma^{\prime}{ }_{n}\left\|v_{n}-S_{i(n)}^{j(n)} x_{n}\right\| \\
& \leq e_{j(n)}\left\|x_{n}-f\right\|+\xi_{j(n)}+\gamma^{\prime}{ }_{n}\left\|v_{n}-S_{i(n)}^{j(n)} x_{n}\right\| .
\end{aligned}
$$

which in turn imply that

$$
\limsup _{n \rightarrow \infty}\left\|x_{n}-f+\gamma_{n}^{\prime}\left(v_{n}-S_{i(n)}^{j(n)} x_{n}\right)\right\| \leq d
$$

and

$$
\limsup _{n \rightarrow \infty}\left\|S_{i(n)}^{j(n)} x_{n}-f+\gamma_{n}^{\prime}\left(v_{n}-S_{i(n)}^{j(n)} x_{n}\right)\right\| \leq d .
$$

On the other hand, we have

$$
\begin{aligned}
\left\|x_{n}-f\right\| & =\left\|\alpha_{n} x_{n-1}+\beta_{n} T_{i(n)}^{j(n)} y_{n}+\gamma_{n} u_{n}-f\right\| \\
& \leq \alpha_{n}\left\|x_{n-1}-f\right\|+\beta_{n}\left\|T_{i(n)}^{j(n)} y_{n}-f\right\|+\gamma_{n}\left\|u_{n}-f\right\| \\
& \leq \alpha_{n}\left\|x_{n-1}-T_{i(n)}^{k(n)} y_{n}\right\|+\left\|T_{i(n)}^{j(n)} y_{n}-f\right\|+\gamma_{n}\left\|u_{n}-f\right\| \\
& \leq \alpha_{n}\left\|x_{n-1}-T_{i(n)}^{k(n)} y_{n}\right\|+e_{j(n)}\left\|y_{n}-f\right\|+\xi_{j(n)}+\gamma_{n}\left\|u_{n}-f\right\|,
\end{aligned}
$$


from which it follows that $\lim \inf _{n \rightarrow \infty}\left\|y_{n}-f\right\| \geq d$. In view of (3.1), we see that lim $\sup _{n \rightarrow \infty}|| y_{n}-f|| \leq d$. This proves that

$$
\lim _{n \rightarrow \infty}\left\|y_{n}-f\right\|=d \text {. }
$$

Notice that

$$
\begin{aligned}
\lim _{n \rightarrow \infty}\left\|y_{n}-f\right\|= & \lim _{n \rightarrow \infty} \| \alpha^{\prime}{ }_{n}\left(x_{n}-f+\gamma^{\prime}{ }_{n}\left(v_{n}-S_{i(n)}^{j(n)} x_{n}\right)\right) \\
& +\left(1-\alpha^{\prime}{ }_{n}\right)\left(S_{i(n)}^{j(n)} x_{n}-f+\gamma^{\prime}{ }_{n}\left(v_{n}-S_{i(n)}^{j(n)} x_{n}\right)\right) \| .
\end{aligned}
$$

This implies from Lemma 2.2 that

$$
\lim _{n \rightarrow \infty}\left\|S_{i(n)}^{j(n)} x_{n}-x_{n}\right\|=0 .
$$

On the other hand, we have

$$
\begin{aligned}
& \left\|T_{i(n)}^{j(n)} x_{n}-x_{n}\right\| \\
& \leq\left\|T_{i(n)}^{j(n)} x_{n}-T_{i(n)}^{j(n)} y_{n}\right\|+\left\|T_{i(n)}^{j(n)} y_{n}-x_{n-1}\right\|+\left\|x_{n-1}-x_{n}\right\| \\
& \leq\left\|T_{i(n)}^{j(n)} x_{n}-T_{i(n)}^{j(n)} y_{n}\right\|+\left\|T_{i(n)}^{j(n)} y_{n}-x_{n-1}\right\|+\left\|x_{n-1}-x_{n}\right\| \\
& \leq L\left\|x_{n}-y_{n}\right\|+\left\|T_{i(n)}^{j(n)} y_{n}-x_{n-1}\right\|+\left\|x_{n-1}-x_{n}\right\| \\
& \leq L \beta^{\prime}{ }_{n}\left\|S_{i(n)}^{j(n)} x_{n}-x_{n}\right\|+L{\gamma^{\prime}}_{n}\left\|v_{n}-x_{n}\right\|+\left\|T_{i(n)}^{j(n)} y_{n}-x_{n-1}\right\| \\
& \quad+\left\|x_{n-1}-x_{n}\right\| .
\end{aligned}
$$

This combines with (3.4), (3.5), and (3.7) gives that

$$
\lim _{n \rightarrow \infty}\left\|T_{i(n)}^{j(n)} x_{n}-x_{n}\right\|=0 .
$$

Since $n=(j(n)-1) N+i(n)$, where $i(n) \in\{1,2, \ldots, N\}$, we see that

$$
\begin{aligned}
\left\|x_{n}-S_{i(n)} x_{n}\right\| & \leq\left\|x_{n}-S_{i(n)}^{j(n)} x_{n}\right\|+\left\|S_{i(n)}^{j(n)} x_{n}-S_{i(n)} x_{n}\right\| \\
& \leq\left\|x_{n}-S_{i(n)}^{j(n)} x_{n}\right\|+L\left\|S_{i(n)}^{j(n)-1} x_{n}-x_{n}\right\| \\
& \leq\left\|x_{n}-S_{i(n)}^{j(n)} x_{n}\right\|+L\left(\left\|S_{i(n)}^{j(n)-1} x_{n}-S_{i(n-N)}^{j(n)-1} x_{n-N}\right\|\right. \\
& \left.+\left\|S_{i(n-N)}^{j(n)-1} x_{n-N}-x_{n-N}\right\|+\left\|x_{n-N}-x_{n}\right\|\right) .
\end{aligned}
$$

Notice that

$$
j(n-N)=j(n)-1 \quad \text { and } \quad i(n-N)=i(n) .
$$

This in turn implies that

$$
\begin{aligned}
\left\|S_{i(n)}^{j(n)-1} x_{n}-S_{i(n-N)}^{j(n)-1} x_{n-N}\right\| & =\left\|S_{i(n)}^{j(n)-1} x_{n}-S_{i(n)}^{j(n)-1} x_{n-N}\right\| \\
& \leq L\left\|x_{n}-x_{n-N}\right\|
\end{aligned}
$$

and

$$
\left\|S_{i(n-N)}^{j(n)-1} x_{n-N}-x_{n-N}\right\|=\left\|S_{i(n-N)}^{j(n-N)} x_{n-N}-x_{n-N}\right\| .
$$


Substituting (3.10) and (3.11) into (3.9) yields that

$$
\begin{gathered}
\left\|x_{n}-S_{i(n)} x_{n}\right\| \leq\left\|x_{n}-S_{i(n)}^{j(n)} x_{n}\right\|+L\left(L\left\|x_{n}-x_{n-N}\right\|\right. \\
\left.+\left\|S_{i(n-N)}^{j(n-N)} x_{n-N}-x_{n-N}\right\|\right) .
\end{gathered}
$$

It follows from (3.6) and (3.7) that

$$
\lim _{n \rightarrow \infty}\left\|x_{n}-S_{i(n)} x_{n}\right\|=0 .
$$

In particular, we see that

$$
\left\{\begin{array}{c}
\lim _{j \rightarrow \infty}\left\|x_{j N+1}-S_{1} x_{j N+1}\right\|=0 \\
\lim _{j \rightarrow \infty}\left\|x_{j N+2}-S_{2} x_{j N+2}\right\|=0 \\
\quad \vdots \\
\lim _{j \rightarrow \infty}\left\|x_{j N+N}-S_{N} x_{j N+N}\right\|=0
\end{array}\right.
$$

For any $r, s=1,2, \ldots, N$, we obtain that

$$
\begin{aligned}
& \left\|x_{j N+s}-S_{r} x_{j N+s}\right\| \\
& \leq\left\|x_{j N+s}-x_{j N+r}\right\|+\left\|x_{j N+r}-S_{r} x_{j N+r}\right\|+\left\|S_{r} x_{j N+r}-S_{r} x_{j N+s}\right\| \\
& \leq(1+L)\left\|x_{j N+s}-x_{j N+r}\right\|+\left\|x_{j N+r}-S_{r} x_{j N+r}\right\| .
\end{aligned}
$$

Letting $j \rightarrow \infty$, we arrive at

$$
\lim _{j \rightarrow \infty}\left\|x_{j N+s}-S_{r} x_{j N+s}\right\|=0,
$$

which is equivalent to

$$
\lim _{n \rightarrow \infty}\left\|x_{n}-S_{r} x_{n}\right\|=0 .
$$

Notice that

$$
\begin{aligned}
\left\|x_{n}-T_{i(n)} x_{n}\right\| & \leq\left\|x_{n}-T_{i(n)}^{j(n)} x_{n}\right\|+\left\|T_{i(n)}^{j(n)} x_{n}-T_{i(n)} x_{n}\right\| \\
& \leq\left\|x_{n}-T_{i(n)}^{j(n)} x_{n}\right\|+L\left\|T_{i(n)}^{j(n)-1} x_{n}-x_{n}\right\| \\
& \leq\left\|x_{n}-T_{i(n)}^{j(n)} x_{n}\right\|+L\left(\left\|T_{i(n)}^{j(n)-1} x_{n}-T_{i(n-N)}^{j(n)-1} x_{n-N}\right\|\right. \\
& \left.+\left\|T_{i(n-N)}^{j(n)-1} x_{n-N}-x_{n-N}\right\|+\left\|x_{n-N}-x_{n}\right\|\right) .
\end{aligned}
$$

On the other hand, we have

$$
\begin{aligned}
\left\|T_{i(n)}^{\mathrm{j}(n)-1} x_{n}-T_{i(n-N)}^{\mathrm{j}(n)-1} x_{n-N}\right\| & =\left\|T_{i(n)}^{\mathrm{j}(n)-1} x_{n}-T_{i(n)}^{\mathrm{j}(n)-1} x_{n-N}\right\| \\
& \leq L\left\|x_{n}-x_{n-N}\right\|
\end{aligned}
$$

and

$$
\left\|T_{i(n-N)}^{j(n)-1} x_{n-N}-x_{n-N}\right\|=\left\|T_{i(n-N)}^{j(n-N)} x_{n-N}-x_{n-N}\right\| .
$$

Substituting (3.15) and (3.16) into (3.14) yields that

$$
\begin{gathered}
\left\|x_{n}-T_{i(n)} x_{n}\right\| \leq\left\|x_{n}-T_{i(n)}^{k(n)} x_{n}\right\|+L\left(L\left\|x_{n}-x_{n-N}\right\|\right. \\
\left.+\left\|T_{i(n-N)}^{k(n-N)} x_{n-N}-x_{n-N}\right\|\right) .
\end{gathered}
$$

It follows from (3.6) and (3.8) that

$$
\lim _{n \rightarrow \infty}\left\|x_{n}-T_{i(n)} x_{n}\right\|=0 .
$$


In particular, we see that

$$
\left\{\begin{array}{c}
\lim _{k \rightarrow \infty}\left\|x_{j N+1}-T_{1} x_{j N+1}\right\|=0 \\
\lim _{k \rightarrow \infty}\left\|x_{j N+2}-T_{2} x_{j N+2}\right\|=0 \\
\vdots \\
\lim _{k \rightarrow \infty}\left\|x_{j N+N}-T_{N} x_{j N+N}\right\|=0 .
\end{array}\right.
$$

For any $r, s=1,2, \ldots, N$, we obtain that

$$
\begin{aligned}
& \left\|x_{j N+s}-T_{r} x_{j N+s}\right\| \\
& \leq\left\|x_{j N+s}-x_{j N+r}\right\|+\left\|x_{j N+r}-T_{r} x_{j N+r}\right\|+\left\|T_{r} x_{j N+r}-T_{r} x_{j N+s}\right\| \\
& \leq(1+L)\left\|x_{j N+s}-x_{j N+r}\right\|+\left\|x_{j N+r}-T_{r} x_{j N+r}\right\| .
\end{aligned}
$$

Letting $j \rightarrow \infty$, we arrive

$$
\lim _{j \rightarrow \infty}\left\|x_{j N+s}-T_{r} x_{j N+s}\right\|=0,
$$

which is equivalent to

$$
\lim _{n \rightarrow \infty}\left\|x_{n}-T_{r} x_{n}\right\|=0 .
$$

This completes the proof. $\square$

Now, we are in a position to give weak convergence theorems.

Theorem 3.2. Let $E$ be a real Hilbert space and $C$ be a nonempty closed convex subset of $E$. Let $T_{i}: C \rightarrow C$ be a uniformly $L_{t}^{i}$-Lipschitz and generalized asymptotically nonexpansive mapping with a sequence $\left\{h_{n}^{i}\right\} \subset[1, \infty)$, where $h_{n}^{i} \rightarrow 1$ as $n \rightarrow \infty$ and $S_{i}: C \rightarrow C$ be a uniformly $L_{s}^{i}$-Lipschitz and generalized asymptotically nonexpansive mapping with a sequence $\left\{k_{n}^{i}\right\} \subset[1, \infty)$, where $k_{n}^{i} \rightarrow 1$ as $n \rightarrow \infty$ for each $1 \leq i \leq N$. Assume that $\mathcal{F}=\bigcap_{i=1}^{N} F\left(T_{i}\right) \bigcap \bigcap_{i=1}^{N} F\left(S_{i}\right) \neq \emptyset$. Let $\left\{u_{n}\right\},\left\{v_{n}\right\}$ be bounded sequences in $C$ and $e_{n}=\max$ $\left\{h_{n}, k_{n}\right\}$, where $h_{n}=\sup \left\{h_{n}^{i}: 1 \leq i \leq N\right\}$ and $k_{n}=\sup \left\{k_{n}^{i}: 1 \leq i \leq N\right\}$. Let $\left\{\alpha_{n}\right\},\left\{\beta_{n}\right\},\left\{\gamma_{n}\right\}$, $\left\{\beta_{n}^{\prime}\right\},\left\{\beta_{n}^{\prime}\right\}$ and $\left\{\gamma_{n}^{\prime}\right\}$ be sequences in $[0,1]$ such that $\alpha_{n}+\beta_{n}+\gamma_{n}=\alpha_{n}^{\prime}+\beta_{n}^{\prime}+\gamma_{n}^{\prime}=1$ for each $n$ $\geq$ 1. Let $\left\{x_{n}\right\}$ be a sequence generated in (2.4). Put $v_{n}^{i}=\max \left\{0, \sup _{x, y \in C}\left(\left\|S_{i}^{n} x-S_{i}^{n} y\right\|-k_{n}^{i}\|x-y\|\right)\right\}$ and $v_{n}^{i}=\max \left\{0, \sup _{x, y \in C}\left(\left\|S_{i}^{n} x-S_{i}^{n} y\right\|-k_{n}^{i}\|x-y\|\right)\right\}$. Let $\xi_{n}=\max \left\{\mu_{n}, v_{n}\right\}$, where $v_{n}=\max \left\{v_{n}^{i}: 1 \leq i \leq N\right\}$ and $v_{n}=\max \left\{v_{n}^{i}: 1 \leq i \leq N\right\}$. Assume that the following restrictions are satisfied:

(a) $\sum_{n=1}^{\infty} \gamma_{n}<\infty$ and $\sum_{n=1}^{\infty} \gamma_{n}^{\prime}<\infty$;

(b) $\sum_{n=1}^{\infty}\left(e_{n}-1\right)<\infty$ and $\sum_{n=1}^{\infty} \xi_{n}<\infty$;

(c) $\beta_{n} L\left(\alpha_{n}^{\prime}+\beta_{n}^{\prime} L\right)<1$, where $L$ is defined in (2.5);

(d) there exist constants $\lambda, \eta \in(0,1)$ such that $\lambda \leq \alpha_{n}, \alpha_{n}^{\prime} \leq \eta$.

Then the sequence $\left\{x_{n}\right\}$ converges weakly to some point in $\mathcal{F}$.

Proof. Since $E$ is a Hilbert space and $\left\{x_{n}\right\}$ is bounded, we can obtain that there exists a subsequence $\left\{x_{n_{p}}\right\}$ of $\left\{x_{n}\right\}$ converges weakly to $x^{*} \in C$. It follows from (3.13) and (3.18) that

$$
\lim _{p \rightarrow \infty}\left\|x_{n_{p}}-T_{r} x_{n_{p}}\right\|=\lim _{p \rightarrow \infty}\left\|x_{n_{p}}-S_{r} x_{n_{p}}\right\|=0 .
$$


Since $I-S_{r}$ and $I-T_{r}$ are demiclosed at origin by Lemma 2.3, we see that $x^{*} \in \mathcal{F}$. Next, we show that the whole sequence $\left\{x_{n}\right\}$ converges weakly to $x^{*}$. Suppose the contrary. Then there exists some subsequence $\left\{x_{n_{q}}\right\}$ of $\left\{x_{n}\right\}$ such that $\left\{x_{n_{q}}\right\}$ converges weakly to $x^{* *} \in C$. In the same way, we can show that $x^{* *} \in \mathcal{F}$. Notice that we have proved that $\lim _{n \rightarrow \infty}|| x_{n}-f \|$ exists for each $f \in \mathcal{F}$. By virtue of Opial's condition of $E$, we have

$$
\begin{aligned}
\liminf _{p \rightarrow \infty}\left\|x_{n_{p}}-x^{*}\right\| & <\liminf _{p \rightarrow \infty}\left\|x_{n_{p}}-x^{* *}\right\| \\
& =\liminf _{q \rightarrow \infty}\left\|x_{n_{q}}-x^{* *}\right\| \\
& <\liminf _{q \rightarrow \infty}\left\|x_{n_{q}}-x^{*}\right\| .
\end{aligned}
$$

This is a contradiction. Hence, $x^{*}=x^{* * *}$. This completes the proof. $\square$

Remark 3.3. Theorem 3.2 which includes the corresponding results announced in Chang et al. [6], Chidume and Shahzad [7], Guo and Cho [10], Plubtieng et al. [14], Qin et al. [15], Thakur [21], Thianwan and Suantai [22], Xu and Ori [23], and Zhou and Chang [24] as special cases mainly improves the results of Cianciaruso et al. [9] in the following aspects.

(1) Extend the mappings from one family of mappings to two families of mappings;

(2) Extend the mappings from the class of asymptotically nonexpansive mappings to the class of generalized asymptotically nonexpansive mappings.

If $S_{r}=I$ for each $r \in\{1,2, \ldots, N\}$ and $\gamma_{n}^{\prime}=0$, then Theorem 3.2 is reduced to the following.

Corollary 3.4. Let $E$ be a real Hilbert space and $C$ be a nonempty closed convex subset of $E$. Let $T_{i}: C \rightarrow C$ be a uniformly $L_{t}^{i}$-Lipschitz and generalized asymptotically nonexpansive mapping with a sequence $\left\{h_{n}^{i}\right\} \subset[1, \infty)$, where $h_{n}^{i} \rightarrow 1$ as $n \rightarrow \infty$ for each $1 \leq$ $i \leq N$. Assume that $\mathcal{F}=\bigcap_{i=1}^{N} F\left(T_{i}\right) \neq \emptyset$. Let $\left\{u_{n}\right\}$ be a bounded sequence in $C$ and $h_{n}=\sup \left\{h_{n}^{i}: 1 \leq i \leq N\right\}$. Let $\left\{\alpha_{n}\right\},\left\{\beta_{n}\right\}$ and $\left\{\gamma_{n}\right\}$ be sequences in $[0,1]$ such that $\alpha_{n}+\beta_{n}$ $+\gamma_{n}=1$ for each $n \geq 1$. Let $\left\{x_{n}\right\}$ be a sequence generated in the following process:

$$
x_{0} \in C, \quad x_{n}=\alpha_{n} x_{n-1}+\beta_{n} T_{i(n)}^{\mathrm{j}(n)} x_{n}+\gamma_{n} u_{n}, \quad n \geq 1 .
$$

Put

$$
\mu_{n}^{i}=\max \left\{0, \sup _{x, y \in C}\left(\left\|T_{i}^{n} x-T_{i}^{n} y\right\|-h_{n}^{i}\|x-y\|\right)\right\} .
$$

$\mu_{n}=\max \left\{\mu_{n}^{i}: 1 \leq i \leq N\right\}$. Assume that the following restrictions are satisfied:

(a) $\sum_{n=1}^{\infty} \gamma_{n}<\infty$;

(b) $\sum_{n=1}^{\infty}\left(h_{n}-1\right)<\infty$ and $\sum_{n=1}^{\infty} \mu_{n}<\infty$;

(c) $\beta_{n} L<1$, where $L=\max \left\{L_{t}^{i}: 1 \leq i \leq N\right\}$;

(d) there exist constants $\lambda, \eta \in(0,1)$ such that $\lambda \geq \alpha_{n}, \alpha_{n}^{\prime} \leq \eta$.

Then the sequence $\left\{x_{n}\right\}$ converges weakly to some point in $\mathcal{F}$.

Next, we are in a position to state strong convergence theorems in a Banach space.

Theorem 3.5. Let $E$ be a real uniformly convex Banach space and $C$ be a nonempty closed convex subset of $E$. Let $T_{i}: C \rightarrow C$ be a uniformly $L_{t}^{i}$-Lipschitz and generalized 
asymptotically nonexpansive mapping with a sequence $\left\{h_{n}^{i}\right\} \subset[1, \infty)$, where $h_{n}^{i} \rightarrow 1$ as $n$ $\rightarrow \infty$ and $S_{i}: C \rightarrow C$ be a uniformly $L_{s}^{i}$-Lipschitz and generalized asymptotically nonexpansive mapping with a sequence $\left\{k_{n}^{i}\right\} \subset[1, \infty)$, where $k_{n}^{i} \rightarrow 1$ as $n \rightarrow \infty$ for each $1 \leq i$ $\leq N$. Assume that $\mathcal{F}=\bigcap_{i=1}^{N} F\left(T_{i}\right) \bigcap \bigcap_{i=1}^{N} F\left(S_{i}\right) \neq \emptyset$. Let $\left\{u_{n}\right\},\left\{v_{n}\right\}$ be bounded sequences in $C$ and $e_{n}=\max \left\{h_{n}, k_{n}\right\}$, where $h_{n}=\sup \left\{h_{n}^{i}: 1 \leq i \leq N\right\}$ and $k_{n}=\sup \left\{k_{n}^{i}: 1 \leq i \leq N\right\}$. Let $\left\{\alpha_{n}\right\},\left\{\beta_{n}\right\},\left\{\gamma_{n}\right\},\left\{\alpha_{n}^{\prime}\right\},\left\{\beta_{n}^{\prime}\right\}$ and $\left\{\gamma_{n}^{\prime}\right\}$ be sequences in $[0,1]$ such that $\alpha_{n}+\beta_{n}+\gamma_{n}=\alpha_{n}^{\prime}+\beta_{n}^{\prime}+\gamma_{n}^{\prime}=1$ for each $n \geq 1$. Let $\left\{x_{n}\right\}$ be a sequence generated in (2.4). Put $\quad \mu_{n}^{i}=\max \left\{0, \sup _{x, y \in C}\left(\left\|T_{i}^{n} x-T_{i}^{n} y\right\|-h_{n}^{i}\|x-y\|\right)\right\}$ and $v_{n}^{i}=\max \left\{0, \sup _{x, y \in C}\left(\left\|S_{i}^{n} x-S_{i}^{n} y\right\|-k_{n}^{i}\|x-y\|\right)\right\}$. Let $\xi_{n}=\max \left\{\mu_{n}, v_{n}\right\}$, where $v_{n}=\max \left\{v_{n}^{i}: 1 \leq i \leq N\right\}$ and $v_{n}=\max \left\{v_{n}^{i}: 1 \leq i \leq N\right\}$. Assume that the following restrictions are satisfied:

(a) $\sum_{n=1}^{\infty} \gamma_{n}<\infty$ and $\sum_{n=1}^{\infty} \gamma_{n}^{\prime}<\infty$;

(b) $\sum_{n=1}^{\infty}\left(e_{n}-1\right)<\infty$ and $\sum_{n=1}^{\infty} \xi_{n}<\infty$;

(c) $\beta_{n} L\left(\alpha_{n}^{\prime}+\beta_{n}^{\prime} L\right)<1$, where $L$ is defined in (2.5);

(d) there exist constants $\lambda, \eta \in(0,1)$ such that $\lambda \geq \alpha_{n}, \alpha_{n}^{\prime} \leq \eta$.

If one of mappings in $\left\{T_{1}, T_{2}, \ldots, T_{N}\right\}$ or one of mappings in $\left\{S_{1}, S_{2}, \ldots, S_{N}\right\}$ are semicompact, then the sequence $\left\{x_{n}\right\}$ converges strongly to some point in $\mathcal{F}$.

Proof. Without loss of generality, we may assume that $S_{1}$ are semicompact. It follows from (3.13) that

$$
\lim _{n \rightarrow \infty}\left\|x_{n}-S_{1} x_{n}\right\|=0 .
$$

By the semicompactness of $S_{1}$, we see that there exists a subsequence $\left\{x_{n_{p}}\right\}$ of $\left\{x_{n}\right\}$ such that $x_{n_{p}} \rightarrow w \in C$ strongly. From (3.13) and (3.18), we have

$$
\left\|w-S_{r} w\right\| \leq\left\|w-x_{n_{p}}\right\|+\left\|x_{n_{p}}-S_{r} x_{n_{p}}\right\|+\left\|S_{r} x_{n_{p}}-S_{r} w\right\|
$$

and

$$
\left\|w-T_{r} w\right\| \leq\left\|w-x_{n_{p}}\right\|+\left\|x_{n_{p}}-T_{r} x_{n_{p}}\right\|+\left\|T_{r} x_{n_{p}}-T_{r} w\right\|
$$

Since $S_{r}$ and $T_{r}$ are Lipshcitz continuous, we obtain that $w \in \mathcal{F}$. From Theorem 3.1, we know that $\lim _{n \rightarrow \infty}|| x_{n}-f \|$ exists for each $f \in \mathcal{F}$. That is, $\lim _{n \rightarrow \infty}|| x_{n}-w \|$ exists. From $x_{n_{p}} \rightarrow w$, we have

$$
\lim _{n \rightarrow \infty}\left\|x_{n}-w\right\|=0 .
$$

This completes the proof of Theorem 3.5. $\square$

If $S_{r}=I$ for each $r \in\{1,2, \ldots, N\}$ and $\gamma_{n}^{\prime}=0$, then Theorem 3.5 is reduced to the following.

Corollary 3.6. Let $E$ be a real uniformly convex Banach space and $C$ be a nonempty closed convex subset of E. Let $T_{i}: C \rightarrow C$ be a uniformly $L_{t}^{i}$ Lipschitz and generalized asymptotically nonexpansive mapping with a sequence $\left\{h_{n}^{i}\right\} \subset[1, \infty)$, where $h_{n}^{i} \rightarrow 1$ as $n$ $\rightarrow \infty$ for each $1 \leq i \leq N$. Assume that $\mathcal{F}=\bigcap_{i=1}^{N} F\left(T_{i}\right) \neq \emptyset$. Let $\left\{u_{n}\right\}$ be a bounded sequence in $C$ and $h_{n}=\sup \left\{h_{n}^{i}: 1 \leq i \leq N\right\}$. Let $\left\{\alpha_{n}\right\},\left\{\beta_{n}\right\}$ and $\left\{\gamma_{n}\right\}$ be sequences in $[0,1]$ such that $\alpha_{n}+\beta_{n}+\gamma_{n}=1$ for each $n \geq 1$. Let $\left\{x_{n}\right\}$ be a sequence generated in 
(3.19). Put $\quad \mu_{n}^{i}=\max \left\{0, \sup _{x, y \in C}\left(\left\|T_{i}^{n} x-T_{i}^{n} y\right\|-h_{n}^{i}\|x-y\|\right)\right\}$.

$\mu_{n}=\max \left\{\mu_{n}^{i}: 1 \leq i \leq N\right\}$. Assume that the following restrictions are satisfied:
(a) $\sum_{n=1}^{\infty} \gamma_{n}<\infty$;
(b) $\sum_{n=1}^{\infty}\left(h_{n}-1\right)<\infty$ and $\sum_{n=1}^{\infty} \mu_{n}<\infty$;
(c) $\beta_{n} L<1$, where $L=\max \left\{L_{t}^{i}: 1 \leq i \leq N\right\}$;
(d) there exist constants $\lambda, \eta \in(0,1)$ such that $\lambda \geq \alpha_{\mathrm{n}}, \alpha_{n}^{\prime} \leq \eta$.

If one of mappings in $\left\{T_{1}, T_{2}, \ldots, T_{N}\right\}$ is semicompact, then the sequence $\left\{x_{n}\right\}$ converges strongly to some point in $\mathcal{F}$.

Theorem 3.7. Let $E$ be a real uniformly convex Banach space and $C$ be a nonempty closed convex subset of $E$. Let $T_{i}: C \rightarrow C$ be a uniformly $L_{t}^{i}$ Lipschitz and generalized asymptotically nonexpansive mapping with a sequence $\left\{h_{n}^{i}\right\} \subset[1, \infty)$, where $h_{n}^{i} \rightarrow 1$ as $n$ $\rightarrow \infty$ and $S_{i}: C \rightarrow C$ be a uniformly $L_{s}^{i}$-Lipschitz and generalized asymptotically nonexpansive mapping with a sequence $\left\{k_{n}^{i}\right\} \subset[1, \infty)$, where $k_{n}^{i} \rightarrow 1$ as $n \rightarrow \infty$ for each $1 \leq i$ $\leq N$. Assume that $\mathcal{F}=\bigcap_{i=1}^{N} F\left(T_{i}\right) \bigcap \bigcap_{i=1}^{N} F\left(S_{i}\right) \neq \emptyset$. Let $\left\{u_{n}\right\},\left\{v_{n}\right\}$ be bounded sequences in $C$ and $e_{n}=\max \left\{h_{n}, k_{n}\right\}$, where $h_{n}=\sup \left\{h_{n}^{i}: 1 \leq i \leq N\right\}$ and $k_{n}=\sup \left\{k_{n}^{i}: 1 \leq i \leq N\right\}$. Let $\left\{\alpha_{n}\right\},\left\{\beta_{n}\right\},\left\{\gamma_{n}\right\},\left\{\alpha_{n}^{\prime}\right\},\left\{\beta_{n}^{\prime}\right\}$ and $\left\{\gamma_{n}^{\prime}\right\}$ be sequences in $[0,1]$ such that $\alpha_{n}+\beta_{n}+\gamma_{n}=\alpha_{n}^{\prime}+\beta_{n}^{\prime}+\gamma_{n}^{\prime}=1$ for each $n \geq 1$. Let $\left\{x_{n}\right\}$ be a sequence generated in (2.4). Put $\quad \mu_{n}^{i}=\max \left\{0, \sup _{x, y \in C}\left(\left\|T_{i}^{n} x-T_{i}^{n} y\right\|-h_{n}^{i}\|x-y\|\right)\right\}$ and $v_{n}^{i}=\max \left\{0, \sup _{x, y \in C}\left(\left\|S_{i}^{n} x-S_{i}^{n} y\right\|-k_{n}^{i}\|x-y\|\right)\right\}$. Let $\xi_{n}=\max \left\{\mu_{n}, v_{n}\right\}$, where $v_{n}=\max \left\{v_{n}^{i}: 1 \leq i \leq N\right\}$ and $v_{n}=\max \left\{v_{n}^{i}: 1 \leq i \leq N\right\}$. Assume that the following restrictions are satisfied:

(a) $\sum_{n=1}^{\infty} \gamma_{n}<\infty$ and $\sum_{n=1}^{\infty} \gamma^{\prime}{ }_{n}<\infty$;

(b) $\sum_{n=1}^{\infty}\left(e_{n}-1\right)<\infty$ and $\sum_{n=1}^{\infty} \xi_{n}<\infty$;

(c) $\beta_{n} L\left(\alpha_{n}^{\prime}+\beta_{n}^{\prime} L\right)<1$, where $L$ is defined in (2.5);

(d) there exist constants $\lambda, \eta \in(0,1)$ such that $\lambda \geq \alpha_{n}, \alpha_{n}^{\prime} \leq \eta$.

If there exists a nondecreasing function $g:[0, \infty) \rightarrow[0, \infty)$ with $g(0)=0$ and $g(m)>0$ for all $m \in(0, \infty)$ such that

$$
\max _{1 \leq r \leq N}\left\{\left\|x-S_{r} x\right\|\right\}+\max _{1 \leq r \leq N}\left\{\left\|x-T_{r} x\right\|\right\} \geq g(\operatorname{dist}(x, \mathcal{F})), \quad \forall x \in C,
$$

then the sequence $\left\{x_{n}\right\}$ converges strongly to some point in $\mathcal{F}$.

Proof. In view of (3.13) and (3.18) that $g\left(\operatorname{dist}\left(x_{n}, \mathcal{F}\right)\right) \rightarrow 0$, which implies $\operatorname{dist}\left(x_{n}, \mathcal{F}\right) \rightarrow 0$. Next, we show that the sequence $\left\{x_{n}\right\}$ is Cauchy. In view of (3.3), we obtain by putting

$$
a_{n}=\frac{\left(1+M_{1}\right)\left(e_{j(n)}-1\right)}{1-R} \quad \text { and } \quad b_{n}=\frac{\left(1+M_{1}\right) \xi_{j(n)}+M_{1} M_{2} \gamma_{n}^{\prime}+M_{3} \gamma_{n}}{1-R}
$$

that

$$
\left\|x_{n}-f\right\| \leq\left(1+a_{n}\right)\left\|x_{n-1}-f\right\|+b_{n} .
$$


It follows, for any positive integers $m, n$, where $m>n>n_{0}$, that

$$
\left\|x_{m}-p\right\| \leq B\left\|x_{n}-p\right\|+B \sum_{i=n+1}^{\infty} b_{i}+b_{m}
$$

where $B=\exp \left\{\sum_{n=1}^{\infty} a_{n}\right\}$. It follows that

$$
\begin{aligned}
\left\|x_{n}-x_{m}\right\| & \leq\left\|x_{n}-f\right\|+\left\|x_{m}-f\right\| \\
& \leq(1+B)\left\|x_{n}-f\right\|+B \sum_{i=n+1}^{\infty} b_{i}+b_{m} .
\end{aligned}
$$

Taking the infimum over all $f \in \mathcal{F}$, we arrive at

$$
\left\|x_{n}-x_{m}\right\| \leq(1+B) \operatorname{dist}\left(x_{n}, \mathcal{F}\right)+B \sum_{i=n+1}^{\infty} b_{i}+b_{m} .
$$

In view of $\sum_{n=1}^{\infty} b_{n}<\infty$ and $\operatorname{dist}\left(x_{n}, \mathcal{F}\right) \rightarrow 0$, we see that $\left\{x_{n}\right\}$ is a Cauchy sequence in $C$ and so $\left\{x_{n}\right\}$ converges strongly to some $x^{*} \in C$. Since $T_{r}$ and $S_{r}$ are Lipschitz for each $r \in\{1,2, \ldots, N\}$, we see that $\mathcal{F}$ is closed. This in turn implies that $x^{*} \in \mathcal{F}$. This completes the proof. $\square$

If $S_{r}=I$ for each $r \in\{1,2, \ldots, N\}$ and $\gamma_{n}^{\prime}=0$, then Theorem 3.7 is reduced to the following.

Corollary 3.8. Let $E$ be a real uniformly convex Banach space and $C$ be a nonempty closed convex subset of E. Let $T_{i}: C \rightarrow C$ be a uniformly $L_{t}^{i}$-Lipschitz and generalized asymptotically nonexpansive mapping with a sequence $\left\{h_{n}^{i}\right\} \subset[1, \infty)$, where $h_{n}^{i} \rightarrow 1$ as $n$ $\rightarrow \infty$ for each $1 \leq i \leq N$. Assume that $\mathcal{F}=\bigcap_{i=1}^{N} F\left(T_{i}\right) \neq \emptyset$. Let $\left\{u_{n}\right\}$ be a bounded sequence in $C$ and $h_{n}=\sup \left\{h_{n}^{i}: 1 \leq i \leq N\right\}$. Let $\left\{\alpha_{n}\right\},\left\{\beta_{n}\right\}$ and $\left\{\gamma_{n}\right\}$ be sequences in $[0,1]$ such that $\alpha_{n}+\beta_{n}+\gamma_{n}=1$ for each $n \geq 1$. Let $\left\{x_{n}\right\}$ be a sequence generated in

$$
\text { Put } \quad \mu_{n}^{i}=\max \left\{0, \sup _{x, y \in C}\left(\left\|T_{i}^{n} x-T_{i}^{n} y\right\|-h_{n}^{i}\|x-y\|\right)\right\} \text {. }
$$

Let $\mu_{n}=\max \left\{\mu_{n}^{i}: 1 \leq i \leq N\right\}$. Assume that the following restrictions are satisfied:

(a) $\sum_{n=1}^{\infty} \gamma_{n}<\infty$;

(b) $\sum_{n=1}^{\infty}\left(h_{n}-1\right)<\infty$ and $\sum_{n=1}^{\infty} \mu_{n}<\infty$;

(c) $\beta_{n} L<1$, where $L=\max \left\{L_{t}^{i}: 1 \leq i \leq N\right\}$;

(d) there exist constants $\lambda, \eta \in(0,1)$ such that, $\lambda \geq \alpha_{n}, \alpha_{n}^{\prime} \leq \eta$.

If there exists a nondecreasing function $g:[0, \infty) \rightarrow[0, \infty)$ with $g(0)=0$ and $g(m)>0$ for all $m \in(0, \infty)$ such that

$$
\max _{1 \leq r \leq N}\left\{\left\|x-T_{r} x\right\|\right\} \geq g(\operatorname{dist}(x, \mathcal{F})), \quad \forall x \in C,
$$

then the sequence $\left\{x_{n}\right\}$ converges strongly to some point in $\mathcal{F}$. 


\author{
Author details \\ ${ }^{1}$ Department of Mathematics, Texas A\&M University - Kingsville, Kingsville, TX 78363-8202, USA ${ }^{2}$ School of \\ Mathematics and Information Sciences, North China University of Water Resources and Electric Power, Zhengzhou \\ 450011, China ${ }^{3}$ Department of Mathematics and RINS, Gyeongsang National University, Jinju 660-701, Korea
}

\title{
Authors' contributions
}

All authors contributed equally and significantly in writing this paper. All authors read and approved the final manuscript.

\section{Competing interests}

The authors declare that they have no competing interests.

Received: 13 April 2011 Accepted: 27 September 2011 Published: 27 September 2011

\section{References}

1. Izmaelov, AF, Solodov, MV: An active set Newton method for mathematical program with complementary constraints. SIAM J Optim. 19, 1003-1027 (2008). doi:10.1137/070690882

2. Kaufman, DE, Smith, RL, Wunderlich, KE: User-equilibrium properties of fixed points in dynamic traffic assignment. Transportation Res Part C. 6, 1-16 (1998). doi:10.1016/S0968-090X(98)00005-9

3. Kotzer, T, Cohen, N, Shamir, J: Image restoration by an ovel method of parallel projectio onto constraint sets. Optim Lett. 20, 1172-1174 (1995). doi:10.1364/OL.20.001172

4. Bauschke, HH, Borwein, JM: On projection algorithms for solving convex feasibility problems. SIAM Rev. 38, 367-426 (1996). doi:10.1137/S0036144593251710

5. Youla, DC: Mathematical theory of image restoration by the method of convex projections. In: Stark H (ed.) Image Recovery: Theory and Applications. pp. 29-77. Academic Press, Florida, USA (1987)

6. Chang, SS, Tan, KK, Lee, HWJ, Chan, CK: On the convergence of implicit iteration process with error for a finite family of asymptotically nonexpansive mappings. J Math Anal Appl. 313, 273-283 (2006). doi:10.1016/j.jmaa.2005.05.075

7. Chidume, CE, Shahzad, N: Strong convergence of an implicit iteration process for a finite family of nonexpansive mappings. Nonlinear Anal. 62, 1149-1156 (2005). doi:10.1016/j.na.2005.05.002

8. Cho, SY, Kang, SM: Approximation of fixed points of pseudocontraction semigroups based on a viscosity iterative process. Appl Math Lett. 24, 224-228 (2011). doi:10.1016/j.aml.2010.09.008

9. Cianciaruso, F, Marino, G, Wang, X: Weak and strong convergence of the Ishikawa iterative process for a finite family of asymptotically nonexpansive mappings. Appl Math Comput. 216, 3558-3567 (2010). doi:10.1016/j.amc.2010.05.001

10. Guo, W, Cho, YJ: On the strong convergence of the implicit iterative processes with errors for a finite family of asymptotically nonexpansive mappings. Appl Math Lett. 21, 1046-1052 (2008). doi:10.1016/j.aml.2007.07.034

11. Hao, Y, Cho, SY, Qin, X: Some weak convergence theorems for a family of asymptotically nonexpansive nonself mappings. Fixed Point Theory Appl 2010, 11 (2010). Article ID 218573

12. Khan, SH, Yildirim, I, Ozdemir, M: Convergence of an implicit algorithm for two families of nonexpansive mappings. Comput Math Appl. 59, 3084-3091 (2010). doi:10.1016/j.camwa.2010.02.029

13. Kim, JK, Nam, YM, Sim, JY: Convergence theorems of implicit iterative sequences for a finite family of asymptotically quasi-nonexpansive type mappings. Nonlinear Anal. 71, e2839-e2848 (2009). doi:10.1016/..na.2009.06.090

14. Plubtieng, S, Ungchittrakool, K, Wangkeeree, R: Implicit iterations of two finite families for nonexpansive mappings in Banach spaces. Numer Funct Anal Optim. 28, 737-749 (2007). doi:10.1080/01630560701348525

15. Qin, X, Cho, YJ, Shang, M: Convergence analysis of implicit iterative algorithms for asymptotically nonexpansive mappings. Appl Math Comput. 210, 542-550 (2009). doi:10.1016/j.amc.2009.01.018

16. Qin, $\mathrm{X}, \mathrm{Kang}, \mathrm{SM}$, Agarwal, RP: On the convergence of an implicit iterative process for generalized asymptotically quasinonexpansive mappings. Fixed Point Theory Appl 2010, 19 (2010). Article ID 714860

17. Schu, J: Weak and strong convergence of fixed points of asymptotically nonexpansive mappings. Bull Austral Math Soc. 43, 153-159 (1991). doi:10.1017/\$0004972700028884

18. Shzhzad, N, Zegeye, H: Strong convergence of an implicit iteration process for a finite family of genrealized asymptotically quasi-nonexpansive maps. Appl Math Comput. 189, 1058-1065 (2007). doi:10.1016/j.amc.2006.11.152

19. Su, Y, Qin, X: General iteration algorithm and convergence rate optimal model for common fixed points of nonexpansive mappings. Appl Math Comput. 186, 271-278 (2007). doi:10.1016/j.amc.2006.07.101

20. Tan, KK, Xu, HK: Approximating fixed points of nonexpansive mappings by the Ishikawa iterative process. J Math Anal Appl. 178, 301-308 (1993). doi:10.1006/jmaa.1993.1309

21. Thakur, BS: Weak and strong convergence of composite implicit iteration process. Appl Math Comput. 190, 965-997 (2007). doi:10.1016/j.amc.2007.01.101

22. Thianwan, S, Suantai, S: Weak and strong convergence of an implicity iteration process for a finite family of nonexpansive mappings. Sci Math Japon. 66, 221-229 (2007)

23. Xu, HK, Ori, RG: An implicit iteration process for nonexpansive mappings. Numer Funct Anal Optim. 22, 767-773 (2001). doi:10.1081/NFA-100105317

24. Zhou, YY, Chang, SS: Convergence of implicit iterative process for a finite of asymptotically nonexpansive mappings in Banach spaces. Numer Funct Anal Optim. 23, 911-921 (2002). doi:10.1081/NFA-120016276

25. Goebel, K, Kirk, WA: A fixed point theorem for asymptotically nonexpansive mappings. Proc Amer Math Soc. 35, 171-174 (1972). doi:10.1090/50002-9939-1972-0298500-3

26. Bruck, RE, Kuczumow, T, Reich, S: Convergence of iterates of asymptotically nonexpansive mappings in Banach spaces with the uniform opial property. Colloq Math. 65, 169-179 (1993)

27. Kirk, WA: Fixed point theorems for non-Lipschitzian mappings of asymptotically nonexpansive type. Israel J Math. 17, 339-346 (1974). doi:10.1007/BF02757136

28. $\mathrm{Xu}, \mathrm{HK}$ : Existence and convergence for fixed points of mappings of asymptotically nonexpansive type. Nonlinear Anal. 16, 1139-1146 (1991). doi:10.1016/0362-546X(91)90201-B 
29. van Dulst, D: Equivalent norms and the fixed point property for nonexpansive mapping. J Lond Math Soc. 25, 139-144 (1982). doi:10.1112/jms/s2-25.1.139

30. Opial, Z: Weak convergence of the sequence of successive appproximations for nonexpansive mappings. Bull Amer Math Soc. 73, 591-597 (1967). doi:10.1090/50002-9904-1967-11761-0

31. Qin, X, Cho, SY, Kim, JK: Convergence theorems on asymptotically pseudocontractive mappings in the intermediate sense. Fixed Point Theory Appl 2010, 14 (2010). Article ID 186874

32. Sahu, DR, Xu, HK, Yao, JC: Asymptotically strict pseudocontractive mappings in the intermediate sens. Nonlinear Anal. 70, 3502-3511 (2009). doi:10.1016/j.na.2008.07.007

doi:10.1186/1687-1812-2011-58

Cite this article as: Agarwal et al: An implicit iterative algorithm with errors for two families of generalized asymptotically nonexpansive mappings. Fixed Point Theory and Applications 2011 2011:58.

Submit your manuscript to a SpringerOpen ${ }^{\circ}$ journal and benefit from:

- Convenient online submission

- Rigorous peer review

- Immediate publication on acceptance

- Open access: articles freely available online

- High visibility within the field

- Retaining the copyright to your article

Submit your next manuscript at $\gg$ springeropen.com 